\title{
Improved Pregnancy Outcomes in a Prospective Study of Pregnant Women Enrolling in an Antenatal Clinic in Western Kenya
}

\author{
Ibrahim I. Daud1,2, Fredrick O. Opinya1', David Midem¹, Moses Kigani³, \\ Elizabeth A. Bukusi', Zipporah Ng'ang'a ${ }^{2}$, Peter 0. Sumba1, Arlene Dent ${ }^{5}$, \\ Rosemary Rochford ${ }^{*}$ \\ ${ }^{1}$ Center for Global Health Research, Kenya Medical Research Institute, Kisumu, Kenya \\ ${ }^{2}$ College of Health Sciences, Jomo Kenyatta University of Agriculture and Technology, Nairobi, Kenya \\ ${ }^{3}$ Chulaimbo Sub-District Hospital, Kisumu, Kenya \\ ${ }^{4}$ Center for Microbiology Research, Kenya Medical Research Institute, Nairobi, Kenya \\ ${ }^{5}$ Center for Global Health and Diseases, Case Western Reserve University, Cleveland, OH, USA \\ ${ }^{6}$ Department of Microbiology and Immunology, SUNY Upstate Medical University, Syracuse, NY, USA \\ Email: ${ }^{*}$ rochforr@upstate.edu
}

Received 4 September 2014; revised 20 October 2014; accepted 5 November 2014

Academic Editor: Elizabeth Soliday, Washington State University Vancouver, USA

Copyright (C) 2014 by authors and Scientific Research Publishing Inc.

This work is licensed under the Creative Commons Attribution International License (CC BY).

http://creativecommons.org/licenses/by/4.0/

cC) (i) Open Access

\section{Abstract}

In areas of sub-Saharan Africa where malaria is endemic, pregnant women are at a greater risk of malaria than non-pregnant women leading to significant adverse consequences including anemia, intrauterine growth retardation, low birth weight (LBW), and pre-term delivery. The Kenya Ministry of Health adopted Intermittent Preventive Treatment (IPT) and use of insecticide-treated nets (ITN) as a National strategy for malaria prevention in pregnancy. In this report, we evaluated the prevalence of malaria, the anthropometric measures of birth outcomes and the reasons for loss to follow up among pregnant women participating in an ongoing cohort study in Western Kenya. A total of 175 HIV-negative pregnant women enrolled at antenatal clinic of Chulaimbo subDistrict hospital were longitudinally evaluated in a monthly follow-up visits through antenatal visits (up to 4 per mother) and delivery. Thirty three percent and $15 \%$ of the pregnant women were malaria positive by real-time quantitative (Q)-PCR and microscopy respectively at enrolment, while $54 \%$ and $23 \%$ of the pregnant women had malaria by Q-PCR and microscopy respectively at any time during follow-up. Of the enrolled study participants, $65 \%$ delivered at Chulaim-

${ }^{*}$ Corresponding author. 
bo hospital. Overall, 39\% (69) of the pregnant women were lost to follow-up. The major reasons for loss to follow up were relocation from the study area (26\%) and delivery at alternative health facilities (25\%). The mean birth weight of the newborn infants was $3202 \mathrm{~g}$ (range, $2000 \mathrm{~g}-4000 \mathrm{~g}$ ). Only 5.3\% of the infants weighed less than $2500 \mathrm{~g}$ (low birth weight). The mean head circumference was $34 \mathrm{~cm}$ (range, $30 \mathrm{~cm}-39 \mathrm{~cm}$ ) with mean Apgar score (at 10 minutes) \pm S.D. of $9.8 \pm 0.97$. In conclusion, we observed decreased adverse pregnancy outcomes among our study population. We recommend a larger study of all pregnant women attending the Chulaimbo hospital so as to assess whether effectiveness of malaria and anemia control programs lead to improved birth outcomes.

\section{Keywords}

Pregnancy, Birth Outcomes, Malaria, Kenya

\section{Introduction}

Globally, about 358,000 maternal deaths were recorded in 2008. Of these, 99\% $(355,000)$ occurred in the developing world, of which, Africa accounted for slightly more than $50 \%$ of the maternal deaths [1]. In Kenya, the maternal mortality ratio (MMR) is estimated at 488 per 100,000 live births [2]. This high mortality rate is largely attributed to low uptake of antenatal care (ANC) services, where only $47 \%$ of mothers completed the recommended four ANC clinic visits during their pregnancy [2]. In addition, a recent survey in Western Kenya that evaluated the cause of death that occurred between 2003-2008 among women of childbearing age showed 8\% pregnancy-related death, which were attributed to infectious diseases such as malaria and HIV [3].

The health status of a woman before and during pregnancy is a key determinant of pregnancy outcomes, and as such poor nutritional status and exposure to infectious diseases during pregnancy contribute to maternal as well as infant mortality [4]-[6]. Low Birth Weight (LBW) is known to be the most important risk factor and a strong predictor of infant mortality [7] [8]. As a result, women who are underweight, anemic and harboring infections such as malaria during pregnancy are at greater risk of delivering LBW babies [5].

In settings of sub-Saharan Africa where malaria and helminthes infections are endemic and co-infections often occur, pregnant women are particularly susceptible to Plasmodium falciparum and helminthes infections [9] [10], causing significant adverse consequences including anemia, intrauterine growth retardation, low birth weight (LBW), and pre-term delivery [7] [8]. In order to reduce the burden of morbidity and mortality associated with malaria during pregnancy, intermittent preventive treatment of malaria in pregnancy (IPT) - using safe and effective antimalarial drugs - with the aim of eliminating any asymptomatic parasitaemia is recommended [11] [12].

Following the observed beneficial outcome of IPT on maternal anemia and reduction of placental malaria [12] [13], the Kenya Ministry of Health introduced and adopted IPT using sulphadoxine-pyrimethamine (SP) in the second and third trimester of pregnancy as well as use of insecticide-treated nets (ITN) as a National strategy for malaria prevention in pregnancy. This policy has been linked to the delivery of antenatal care. In this report, we evaluated the malaria prevalence, the anthropometric measures of birth outcomes and the reasons for loss to follow up among the pregnant women participating in an ongoing cohort study in Western Kenya, where the foregoing policy is enforced.

\section{Methodology}

This is a prospective descriptive study to investigate the anthropometric measures of birth outcomes, and explore reasons for loss to follow-up among pregnant women participating in a cohort study investigating the persistence and control of Epstein Barr Virus in pregnant women and their infants at Chulaimbo sub-District hospital. Chulaimbo sub-district hospital serves a rural population in Kisumu County. This area is a malaria holoendemic area and has two rainy seasons with the main peak in March-May and the secondary peak in September-November. Details of recruitment and follow up have been previously described [14]. Briefly, during the period from June-November 2011, pregnant women of all gravidities attending the routine antenatal clinics at 
Chulaimbo sub-District Hospital were screened. Inclusion criteria included, gestational age less than 30 weeks, residency within a $10 \mathrm{~km}$ distance of the hospital, willingness to return to the clinic for follow up clinical procedures and laboratory testing, and having a normal hemoglobin level.

All pregnant women were tested for HIV as part of Prevention of Maternal-to-Child-Transmission of HIV programs in accordance with the Kenya Ministry of Health national guidelines. Two hundred pregnant women were screened. Of these 25 were HIV positive and were excluded from further follow up. The remaining 175 were longitudinally evaluated in a monthly antenatal follow-up visits (up to 4 per mother), through delivery. Maternal and child health services are provided in this hospital in accordance with the Kenya Ministry of Health $(\mathrm{MOH})$ policy.

Approval of this study was obtained from both the Kenya Medical Research Institute and SUNY Upstate Medical University Ethical Review Boards. Written informed consent was obtained from the study participants before any sample collection.

\section{Data Collection}

Eligible pregnant women were consented and a pre-designed questionnaire that included socio-demographic data, information on maternal age, gravidity and gestation was completed. Information on medical and obstetric history, past history of malaria, the use of anti-malarial, deworming and herbal drugs was also collected.

Women were scheduled for follow up visits on a monthly basis until delivery. All pregnant women were provided with IPT with sulfadoxine/pyrimethamine (SP), ITN and deworming during pregnancy in accordance with the Kenya Ministry of Health guidelines. The guideline recommends that at least 2 doses of SP should be administered after the first trimester of pregnancy. At delivery, information on state of the newborn (whether alive or stillbirth), weight, length, head circumference, and sex were recorded.

\section{Laboratory Tests}

Detailed description on sample collection, processing and storage has been described previously [14]. Briefly, at enrolment, participants provided 2 - $4 \mathrm{ml}$ of venous blood sample collected in EDTA tubes and measurements of hemoglobin levels were determined using a portable $\beta$-hemoglobin photometer (Hemocue AB Angelholm, Sweden), while complete blood counts were performed with a Beckman coulter AcT diff2 (Beckman-coulter corporations, Miami, FL, USA). Finger prick blood samples were collected at follow-up visits for thick and thin blood films and DNA extraction. Urine and stool samples were obtained and examined for the presence of intestinal helminthes and pathogenic protozoans. Malaria diagnosis by Q-PCR was performed as previously described [15].

\section{Data Analysis}

All statistical analyses were performed using Stata, IC software (13.1, StataCorp LP, College Station, TX, USA). The data are expressed as percentages for categorical data while continuous data are presented using mean with range.

\section{Results}

\subsection{Socio-Demographics, Clinical and Obstetric Characteristics of the Study Participants}

Of the 200 women screened for the study, 25 were HIV positive and were excluded from further follow up. The remaining 175 pregnant women were longitudinally evaluated in monthly follow-up visits through antenatal visits (up to 4 per mother) and delivery. The socio-demographic and obstetric characteristics have been described previously [14]. Briefly, the mean age of the women was 22.3 ( \pm 5.6 S.D.) years, primagravid constituted 39\%, with the majority (58\%) educated to at least upper primary level of education. Thirty three percent $(n=57)$ and $15 \%(n=26)$ of the pregnant women were malaria positive by RTQ-PCR and microscopy respectively at enrolment. In addition, 54\% $(\mathrm{n}=94)$ and $23 \%(\mathrm{n}=40)$ of the pregnant women had malaria by Q-PCR and microscopy respectively, at any time during follow-up.

\subsection{Reasons for Loss to Follow-Up during Pregnancy}

Overall, 39\% (69) of the pregnant women were lost to follow-up. The reasons for loss to follow up were reloca- 
tion from the study area $26 \%$ (18/69), delivery at alternative health facilities $25 \%$ (17/69), withdrawal from the study $19 \%(13 / 69)$, delivery at home $16 \%(11 / 69)$, and other $14 \%(10 / 69)$ (Twin delivery $4 \%(3 / 69)$, spontaneous abortion $2 \%(2 / 69)$, mole pregnancy $1 \%(1 / 69)$, macerated birth $1 \%(1 / 69)$, born with congenital abnormality $1 \%(1 / 69)$, lost contact $1 \%(1 / 69)$, mother died $1 \%(1 / 69)$.

\subsection{Pregnancy Outcomes}

Of the 175 pregnant women enrolled and actively followed monthly, 7\% (13/175) withdrew from study and their data excluded from analysis. Of the remaining 162 pregnant women, 65\% (105/162) delivered at Chulaimbo hospital, 20\% (33/162) delivered at alternative health facilities and 15\% (24/162) delivered at home or on the way to the hospital. The anthropometric measures of birth outcomes were recorded for mothers that delivered at Chulaimbo hospital. The mean birth weight of the newborn infants was $3202 \mathrm{~g}$ (range, $2000 \mathrm{~g}-4000 \mathrm{~g}$ ). Only $5 \%(n=5)$ of the infants weighed less than $2500 \mathrm{~g}$ (low birth weight). The mean head circumference was $34 \mathrm{~cm}$ (range, $30 \mathrm{~cm}-39 \mathrm{~cm}$ ) with mean Apgar score (at 10 minutes) \pm S.D. of $9.8 \pm 0.97$. Of the 5 infants that were LBW, two infants were born to mothers that were malaria positive by both Q-PCR and microscopy at enrolment. Table 1 shows the anthropometric measures of birth outcomes.

\section{Discussion}

In this study, we observed a low prevalence of adverse pregnancy outcomes with only a 5\% prevalence of LBW among the infants that were born to mothers that delivered at Chulaimbo sub-district hospital. Other studies in similar sites have observed LBW outcomes of about 18\% [16] [17]. Risk factors associated with LBW include malaria [18], anemia [17] [19] [20] and late commencement of antenatal care [18] or a combination thereof. Because we recruited the majority (61\%) of our participants when they were in their second trimester of pregnancy [14], it is likely that they have had more ANC visits (average of 4 visits) and therefore more medical evaluation, which could have subsequently lead to an improved birth outcomes. In addition, as part of the study participant's health care, the pregnant women were closely monitored for any illness and all cases were reported to the study clinicians for treatment as per Kenya Ministry of Health (MOH) guidelines.

Pregnant women living in malaria holoendemic areas have an increased risk of malaria during pregnancy coupled with high parasite densities compared to non-pregnant women [21], and pregnant women are often asymptomatic [22]. This means that infected women not attending ANCs frequently remain untreated leading to adverse pregnancy outcomes such as LBW, and anemia [23]. For this particular cohort, however, pregnant women were closely monitored and provided with IPT; this may have reduced the risk of untoward pregnancy outcomes. Furthermore, Chulaimbo hospital has a holistic Maternal and child health services in place in accordance with the Kenya Ministry of Health $(\mathrm{MOH})$ policy. The national strategy for malaria prevention in pregnancy in place may have lead to reduced adverse pregnancy outcomes not only in our cohort but also to all preg-

Table 1. Anthropometric measures of birth outcomes $(n=95)$.

\begin{tabular}{cc}
\hline Characteristics & Summary \\
Sex & \\
Male & $53 \%(50)$ \\
Female & $47 \%(45)$ \\
Axillary temperature $\left({ }^{\circ} \mathrm{C}\right)( \pm$ S.D. $)$ & $35.7(0.78)$ \\
Mean Weight (g) (range) & $3202(2000-4000)$ \\
Mean Length (cm) (range) & $48.5(40-55)$ \\
Mean Head circumference (cm) (range) & $34.9(30-39)$ \\
Mean APGAR score (at 1 minute) $( \pm$ S.D.) & $8.9(1.2)$ \\
Mean APGAR score (at 10 minutes) ( \pm S.D. $)$ & $9.8(0.97)$ \\
Low birth weight & \\
Yes $(<2500$ g) & $5.3 \%(5)$ \\
No $(\geq 2500$ g) & $94.7 \%(90)$ \\
\hline
\end{tabular}


nant women attending this hospital. Of the 5 infants that were LBW, two were born to mothers that had malaria during pregnancy. One pregnant woman had persistent detectable malaria infection by Q-PCR until delivery despite provision of IPT, suggesting that either the malaria parasites were resistant to the antimalarial drugs or she was non-adherent.

Overall, 85\% of the pregnant women enrolled in our study delivered at health facilities, whereas $15 \%$ delivered at home or on their way to the hospital. A previous study from a neighboring area reported that the majority of women (83\%) delivered outside of a health facility [24]. In addition, a recent national survey reported that $43 \%$ of pregnant women deliver at health facility whereas $56 \%$ delivered at home [2], suggesting that our findings of home deliveries are far lower than the national estimate and that of the neighboring area.

A potential limitation of our study is that because we only included and limited our analysis to pregnant women who were enrolled in our cohort study and delivered at Chulaimbo hospital, our findings may not be an exact reflection of the whole study area. Moreover, because we had rather stringent inclusion criteria and deliberately excluded pregnant women with severe anemia and other apparent infections besides malaria, in line with the primary objectives of the parent study, we may therefore have introduced a selection bias and hence our results are probably not representative of all pregnant women in the area.

\section{Conclusion}

In conclusion, we observed decreased adverse pregnancy outcomes among our study population. We recommend a larger study of all pregnant women attending the Chulaimbo hospital with a view to assess whether effectiveness of malaria, intestinal helminthes, and anemia control programs lead to improved birth outcomes.

\section{Acknowledgements}

This work was supported by NIH grant (R01CA102667 and R01AI098511) and D43 Training grant (153707). We thank the study participants and Justus Opondo for technical assistance. This manuscript was approved by the Director Kenya Medical Research Institute for publication.

\section{References}

[1] World Health Organization U, UNFPA and the World Bank (2010) Trends in Maternal Mortality: 1990 to 2010. In Geneva, Switzerland. http://whqlibdoc.who.int/publications/2010/9789241500265 eng.pdf

[2] Macro: KNBoSKaI (2011) Kenya Demographic and Health Survey 2008-09. Calverton, Maryland, USA http://www.measuredhs.com/pubs/pdf/FR229/FR229.pdf

[3] Desai, M., Phillips-Howard, P.A., Odhiambo, F.O., Katana, A., Ouma, P., et al. (2013) An Analysis of Pregnancy-Related Mortality in the KEMRI/CDC Health and Demographic Surveillance System in Western Kenya. PLoS One, 8. http://dx.doi.org/10.1371/journal.pone.0068733

[4] Lartey, A. (2008) Maternal and Child Nutrition in Sub-Saharan Africa: Challenges and Interventions. Proceedings of the Nutrition Society, 67, 105-108. http://dx.doi.org/10.1017/S0029665108006083

[5] Steketee, R.W. (2003) Pregnancy, Nutrition and Parasitic Diseases. Journal of Nutrition, 133, 1661S-1667S.

[6] Abu-Saad, K. and Fraser, D. (2010) Maternal Nutrition and Birth Outcomes. Epidemiologic Reviews, 32, 5-25. http://dx.doi.org/10.1093/epirev/mxq001

[7] Steketee, R.W., Nahlen, B.L., Parise, M.E. and Menendez, C. (2001) The Burden of Malaria in Pregnancy in Malaria-Endemic Areas. The American Journal of Tropical Medicine and Hygiene, 64, 28-35.

[8] Sullivan, A.D., Nyirenda, T., Cullinan, T., Taylor, T., Harlow, S.D., et al. (1999) Malaria Infection during Pregnancy: Intrauterine Growth Retardation and Preterm Delivery in Malawi. The Journal of Infectious Disease, 179, 1580-1583. http://dx.doi.org/10.1086/314752

[9] Brabin, B.J. (1983) An Analysis of Malaria in Pregnancy in Africa. Bulletin of the World Health Organization, 61, 1005-1016.

[10] McGregor, I.A. (1984) Epidemiology, Malaria and Pregnancy. The American Journal of Tropical Medicine and Hygiene, 33, 517-525.

[11] Rogerson, S.J., Chaluluka, E., Kanjala, M., Mkundika, P., Mhango, C. and Molyneux, M.E. (2000) Intermittent Sulfadoxine-Pyrimethamine in Pregnancy: Effectiveness against Malaria Morbidity in Blantyre, Malawi, in 1997-1999. Transactions of the Royal Society of Tropical Medicine and Hygiene, 94, 549-553. http://dx.doi.org/10.1016/S0035-9203(00)90083-X 
[12] Shulman, C.E., Dorman, E.K., Cutts, F., Kawuondo, K., Bulmer, J.N., Peshu, N. and Marsh, K. (1999) Intermittent Sulphadoxine-Pyrimethamine to Prevent Severe Anaemia Secondary to Malaria in Pregnancy: A Randomised PlaceboControlled Trial. Lancet, 353, 632-636. http://dx.doi.org/10.1016/S0140-6736(98)07318-8

[13] Parise, M.E., Ayisi, J.G., Nahlen, B.L., Schultz, L.J., Roberts, J.M., Misore, A., et al. (1998) Efficacy of SulfadoxinePyrimethamine for Prevention of Placental Malaria in an Area of Kenya with a High Prevalence of Malaria and Human Immunodeficiency Virus Infection. American Journal of Tropical Medicine and Hygiene, 59, 813-822.

[14] Daud, I., Ogolla, S., Amolo, A., Namuyenga, E., Simbiri, K., Bukusi, E.A., et al. (2014) Plasmodium falciparum Infection Is Associated with Epstein-Barr Virus Reactivation in Pregnant Women Living in Malaria Holoendemic Area of Western Kenya. Maternal and Child Health Journal, 1-9.

[15] Hermsen, C.C., Telgt, D.S., Linders, E.H., van de Locht, L.A., Eling, W.M., Mensink, E.J.B.M. and Sauerwein, R.W. (2001) Detection of Plasmodium falciparum Malaria Parasites in Vivo by Real-Time Quantitative PCR. Molecular and Biochemical Parasitology, 118, 247-251. http://dx.doi.org/10.1016/S0166-6851(01)00379-6

[16] Menendez, C., Ordi, J., Ismail, M.R., Ventura, P.J., Aponte, J.J., Kahigwa, E., et al. (2000) The Impact of Placental Malaria on Gestational Age and Birth Weight. Journal of Infectious Diseases, 181, 1740-1745. http://dx.doi.org/10.1086/315449

[17] Yatich, N.J., Jolly, P.E., Funkhouser, E., Agbenyega, T., Rayner, J.C., Ehiri, J.E., et al. (2010) The Effect of Malaria and Intestinal Helminth Coinfection on Birth Outcomes in Kumasi, Ghana. American Journal of Tropical Medicine and Hygiene, 82, 28-34. http://dx.doi.org/10.4269/ajtmh.2010.09-0165

[18] Gebremariam, A. (2005) Factors Predisposing to Low Birth Weight in Jimma Hospital South Western Ethiopia. East African Medical Journal, 82, 554-558.

[19] Tako, E.A., Zhou, A., Lohoue, J., Leke, R., Taylor, D.W. and Leke, R.F. (2005) Risk Factors for Placental Malaria and Its Effect on Pregnancy Outcome in Yaounde, Cameroon. American Journal of Tropical Medicine and Hygiene, 72 , 236-242.

[20] Yazdani, M., Tadbiri, M. and Shakeri, S. (2004) Maternal Hemoglobin Level, Prematurity, and Low Birth Weight. International Journal of Gynecology and Obstetrics, 85, 163-164. http://dx.doi.org/10.1016/j.ijgo.2003.07.004

[21] Bouyou-Akotet, M.K., Ionete-Collard, D.E., Mabika-Manfoumbi, M., Kendjo, E., Matsiegui, P.B., et al. (2003) Prevalence of Plasmodium falciparum Infection in Pregnant Women in Gabon. Malaria Journal, 2, 18. http://dx.doi.org/10.1186/1475-2875-2-18

[22] Menendez, C., D’Alessandro, U. and ter Kuile, F.O. (2007) Reducing the Burden of Malaria in Pregnancy by Preventive Strategies. Lancet Infectious Diseases, 7, 126-135. http://dx.doi.org/10.1016/S1473-3099(07)70024-5

[23] Desai, M., ter Kuile, F.O., Nosten, F., McGready, R., Asamoa, K., Brabin, B. and Newman, R.D. (2007) Epidemiology and Burden of Malaria in Pregnancy. Lancet Infectious Diseases, 7, 93-104. http://dx.doi.org/10.1016/S1473-3099(07)70021-X

[24] van Eijk, A.M., Bles, H.M., Odhiambo, F., Ayisi, J.G., Blokland, I.E., Rosen, D.H., et al. (2006) Use of Antenatal Services and Delivery Care among Women in Rural Western Kenya: A Community Based Survey. Reproductive Health, $\mathbf{3}$, 2. http://dx.doi.org/10.1186/1742-4755-3-2 
Scientific Research Publishing (SCIRP) is one of the largest Open Access journal publishers. It is currently publishing more than 200 open access, online, peer-reviewed journals covering a wide range of academic disciplines. SCIRP serves the worldwide academic communities and contributes to the progress and application of science with its publication.

Other selected journals from SCIRP are listed as below. Submit your manuscript to us via either submit@scirp.org or Online Submission Portal.
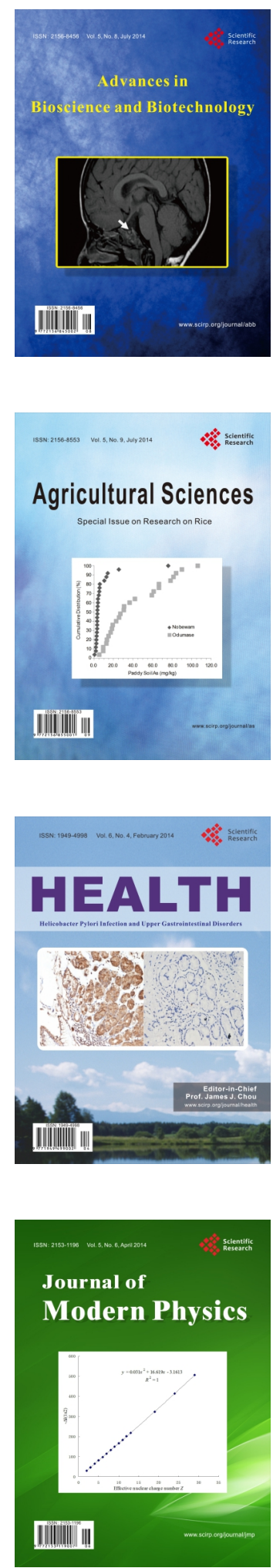
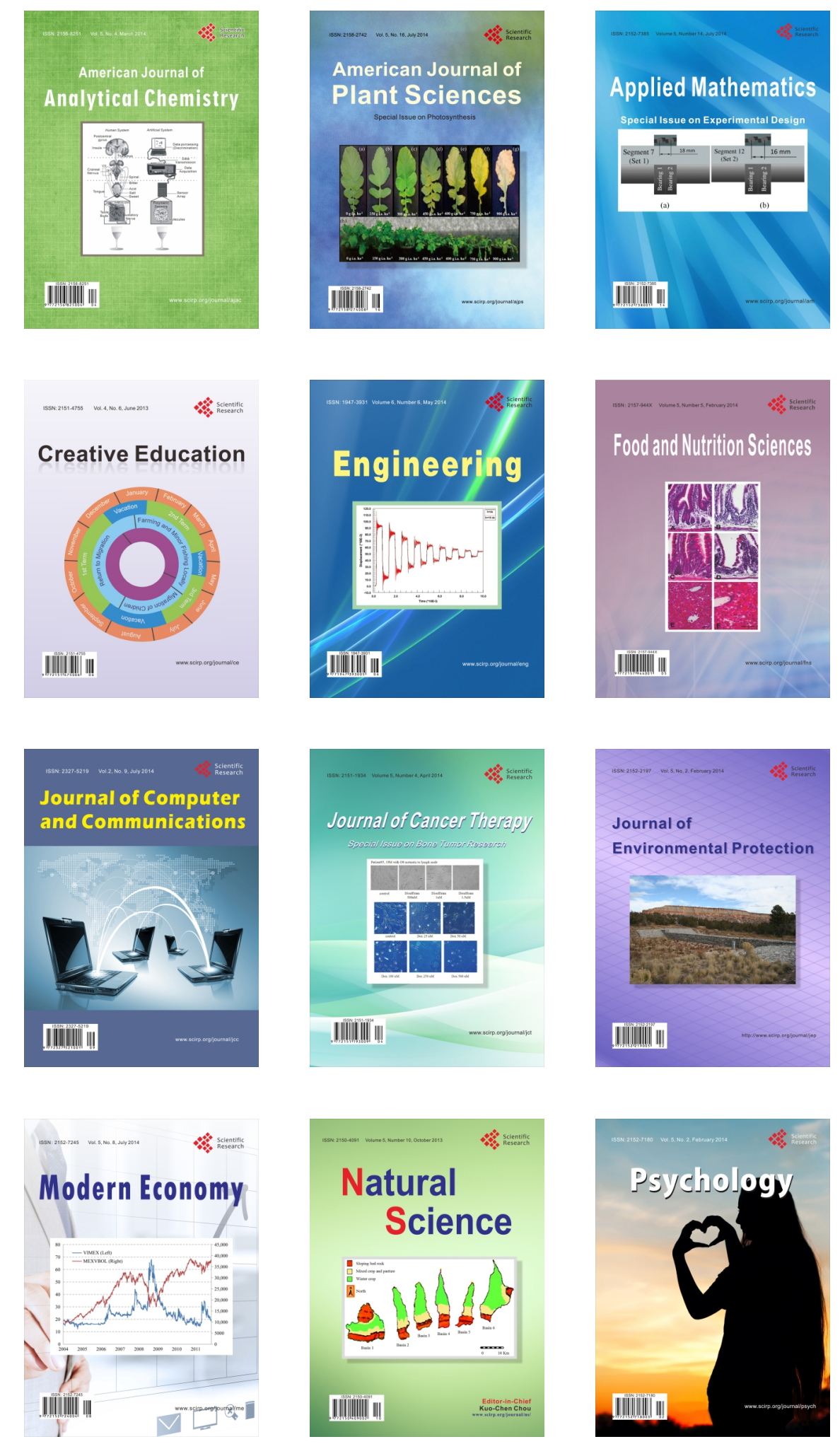